\title{
A survey of mathematical models of human performance using power and energy
}

\author{
Vijay Sarthy M. Sreedhara ${ }^{1}$ (D), Gregory M. Mocko ${ }^{*^{*}}$ (D) and Randolph E. Hutchison ${ }^{2}$ (B)
}

\begin{abstract}
The ability to predict the systematic decrease of power during physical exertion gives valuable insights into health, performance, and injury. This review surveys the research of power-based models of fatigue and recovery within the area of human performance. Upon a thorough review of available literature, it is observed that the twoparameter critical power model is most popular due to its simplicity. This two-parameter model is a hyperbolic relationship between power and time with critical power as the power-asymptote and the curvature constant denoted by $W$. Critical power (CP) is a theoretical power output that can be sustained indefinitely by an individual, and the curvature constant $\left(W^{\prime}\right)$ represents the amount of work that can be done above CP. Different methods and models have been validated to determine CP and $W^{\prime}$, most of which are algebraic manipulations of the twoparameter model. The models yield different $C P$ and $W^{\prime}$ estimates for the same data depending on the regression fit and rounding off approximations. These estimates, at the subject level, have an inherent day-to-day variability called intra-individual variability (IIV) associated with them, which is not captured by any of the existing methods. This calls for a need for new methods to arrive at the IIV associated with CP and W'. Furthermore, existing models focus on the expenditure of $W^{\prime}$ for efforts above CP and do not model its recovery in the sub-CP domain. Thus, there is a need for methods and models that account for (i) the IIV to measure the effectiveness of individual training prescriptions and (ii) the recovery of $W^{\prime}$ to aid human performance optimization.
\end{abstract}

Keywords: Human performance modeling, Fatigue, Time to exhaustion, Energy expenditure, Recovery, Critical power

\section{Key Points}

- Mathematical models of human energy expenditure and recovery present opportunities in quantifying, evaluating, and optimizing performance.

- Established models are focused on energy expenditure and the available models that focus on recovery need refinement to be used in real-time performance optimization.

- Existing models derived from group data neglect the intra-individual variability (IIV) which is critical in evaluating improvements and optimizing performance at the individual level.

\footnotetext{
* Correspondence: gmocko@clemson.edu

${ }^{1}$ Department of Mechanical Engineering, Clemson University, 243 Fluor

Daniel EIB, Clemson, SC 29634-0921, USA

Full list of author information is available at the end of the article
}

\section{Background}

The study of human fatigue and energy expenditure, and to a lesser degree recovery, has been a focal area of research since the early 1900s. Seminal works in the fields of exercise physiology and performance modeling by A. V. Hill [1], Monod and Scherrer [2], and Ward-Smith [3] have laid the groundwork for modeling energy expenditure during prolonged exertion. Recently, researchers have developed formal mathematical models that aid in better management of performance and push limits of human endurance. Most available models have originated from cycle ergometer tests [4] due to the ease of measuring power in cycling and then applied to other forms of exercise like running [5], swimming [6], and rowing [7]. Additionally, most of these models focus on energy exertion with only a few publications that focus on 
energy recovery, which could give us valuable insight into the physiological underpinnings of fatigue, recovery, and ultimately optimizing performance. Furthermore, developing models of human performance and fatigue lead to applications such as mission planning of soldiers and investigating the influence of physical activity on cardiovascular and overall health of a human being.

The purpose of this review is to survey the available literature and summarize all the existing power-based models of human performance. Additionally, this review will identify potential research opportunities to advance the field of human performance in terms of modeling individual variance seen in performance metrics, expenditure and recovery models of work capacity, the potential use of performance models in team sports, the influence of exercise on health, and the use of wearable sensors in mitigating the dependency on laboratory equipment.

\section{Main Text \\ Modeling Performance Using Power}

There are several definitions of fatigue across researchers that limit the ability to measure and develop mathematical models $[8,9]$. For the purpose of this manuscript, fatigue is defined as an exercise induced progressive loss of the ability to sustain maximum power (energy exertion) over a desired duration of time [8-12]. Thus, fatigue is a dynamic process that leads to a drop in the required exercise intensity, which eventually leads to termination of exercise due to exhaustion [13-17]. Exercise intensity is generally categorized as severe, heavy, or moderate [18, 19] based on blood lactate levels [20], maximum oxygen uptake $\left(\dot{\mathrm{V}} \mathrm{O}_{2 \max }\right)[21,22]$, or power output [22]. Maximal lactate steady state (MLSS) is often used to categorize exercise intensity, which is defined as the highest blood lactate concentration that can be maintained without further accumulation during sub-maximal work [23, 24]. The exercise intensity associated with MLSS indicates the highest intensity that can be supported by aerobic mechanisms $[23,25]$. Several methods have been developed to determine MLSS; however, all of them involve taking blood samples and measuring the lactate concentration. Critical power (CP), a theoretical power level which a human can maintain indefinitely [2], is shown to be in close vicinity to the power at which MLSS occurs [26-28]. Moreover, the oxygen uptake $\left(\mathrm{V}_{2}\right)$ and blood lactate levels have been shown to attain a steady state during exercise below CP and hence can be classified as either moderate (below lactate threshold) or heavy (from lactate threshold to CP) intensity [22, 29]. However, exercise above $\mathrm{CP}$ is categorized as severe intensity because $\dot{\mathrm{VO}}_{2}$ and blood lactate levels cannot attain a steady state [22]. Thus, CP represents the boundary between heavy and severe intensity exercises [30] and provides a convenient and non-invasive way of determining exercise intensity [22, 29]. Furthermore, researchers opine that $\mathrm{CP}$ could be the most important fatigue threshold to determine exercise intensity and is the gold-standard to determine the maximal metabolic steady state compared to other parameters such as MLSS, $\% \dot{\mathrm{VO}}_{2}$, lactate threshold (LT), or gas exchange threshold (GET) as it enables population level standardizations $[17,31]$.

The critical power concept was introduced by Monod and Scherrer [2] using a linear relationship between total work done and time-to-exhaustion. Monod and Scherrer's work was based on A. V. Hill's [1] observations pertaining to athletic records in different sports. Monod and Scherrer coined the terms critical power $(\mathrm{CP})$ and limit work $\left(W_{\text {Lim }}\right)$. They defined $\mathrm{CP}$ as the power output that an athlete can generate indefinitely and $W_{\mathrm{Lim}}$ as the total work done until exhaustion at a constant work-rate above $\mathrm{CP}$ related by a linear relationship given by:

$$
W_{\mathrm{Lim}}=a+b \cdot t_{\mathrm{Lim}}
$$

where " $a$ " is an energy reserve in the units of work (Joules) and the constant " $b$ " is the critical power in Watts, and $t_{\text {Lim }}$ is time-to-exhaustion in seconds. Monod and Scherrer derived a hyperbolic form for $t_{\text {Lim }}$ by substituting $W_{\text {Lim }}$ as:

$$
W_{\text {Lim }}=P \cdot t_{\text {Lim }}
$$

Using Eq. 2 and transforming Eq. 1 as:

$$
t_{\text {Lim }}=\frac{a}{P-b}
$$

where $P$ is power in watts. Moritani and colleagues [4] extended the critical power concept to cycling using a series of cycle ergometry tests and called the term " $a$ " as anaerobic reserve deriving the linear relationship between $P$ and $1 / t_{\text {Lim }}$ from Eq. 3 given by:

$$
P=\frac{a}{t_{\text {Lim }}}+b
$$

Whipp and colleagues [32] then fit a hyperbolic curve between $P$ and $t_{\text {Lim }}$ with a time asymptote at a power level that is equal to $\mathrm{CP}$ and denoted the anaerobic reserve term as $W^{\prime}$. The anaerobic reserve term $W^{\prime}$ has since been referred to as anaerobic work capacity (AWC), and these two terms have been used interchangeably. However, it has been shown that $W^{\prime}$ is not equal to AWC and the two terms should not be used 
interchangeably [17, 33]. Additionally, it should be noted that $W^{\prime}$ (pronounced $W$ prime) may lead to confusion in mathematical modeling as it is common notation to use "prime" to represent the first derivative with respect to time. Rewriting Eq. 4 by replacing " $a$ " with $W$ ' and " $b$ " with $\mathrm{CP}$ yields the following relationship:

$$
P=C P+\frac{W^{\prime}}{t_{\text {Lim }}}
$$

Equation 5, widely regarded as the two-parameter model, has been transformed to its linear form, first seen in [4] and later in $[2,34-36]$, by plotting power versus $1 / t_{\text {Lim }}$ with $\mathrm{CP}$ and $W^{\prime}$ representing the $y$-intercept and slope respectively as shown in Fig. 1. The CP concept has been applied to running [5], swimming [6], and rowing [7] with analogous parameters such as critical velocity $(\mathrm{CV})$ and distance capacity $\left(D^{\prime}\right)$ instead of $\mathrm{CP}$ and $W^{\prime}$ respectively.

A limitation of the $\mathrm{CP}$ concept described by Eq. 5 is that as $t_{\mathrm{Lim}}$ approaches $0, P$ tends to infinity (see Fig. 2). This is not realistic as there is a limit to the instantaneous maximum power that a human can produce [37, 38]. Moreover, Josephson [39] states that the maximum power output for a muscle occurs at $30 \%$ of its maximum shortening velocity $\left(V_{\max }\right)$. It takes a short duration of time for the muscle to reach $0.3 V_{\max }$ starting from rest. Therefore, it may beneficial to define the instantaneous maximum power as the average power-output for one crank rotation [40]. Additionally, some

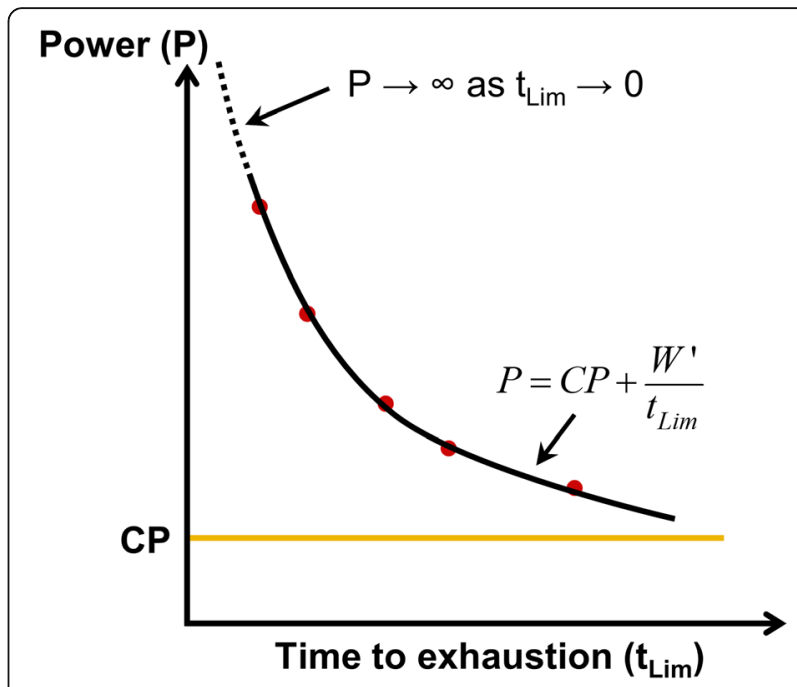

Fig. 2 The two-parameter model and its limitations. As $t_{\text {Lim }}$ tends to $0, P$ tends to $\infty$, and critical power (CP) is the power asymptote at $t_{\text {Lim }}=\infty$

publications have reported that the average duration for which the CP can be maintained is less than an hour [41-44], while others have reported that it can be maintained for approximately over an hour [45, 46]. D. W. Hill [35] suggests that the end point of the tests proposed to the subjects in these studies, i.e., $24-30 \mathrm{~min}$ in $[47,48]$ and $60-90 \mathrm{~min}$ in $[41,45]$ may have influenced the outcome.

Ward-Smith [3] proposed a model to address these limitations that was able to predict sprint performance between $100 \mathrm{~m}$ and $10,000 \mathrm{~m}$. Ward-Smith's
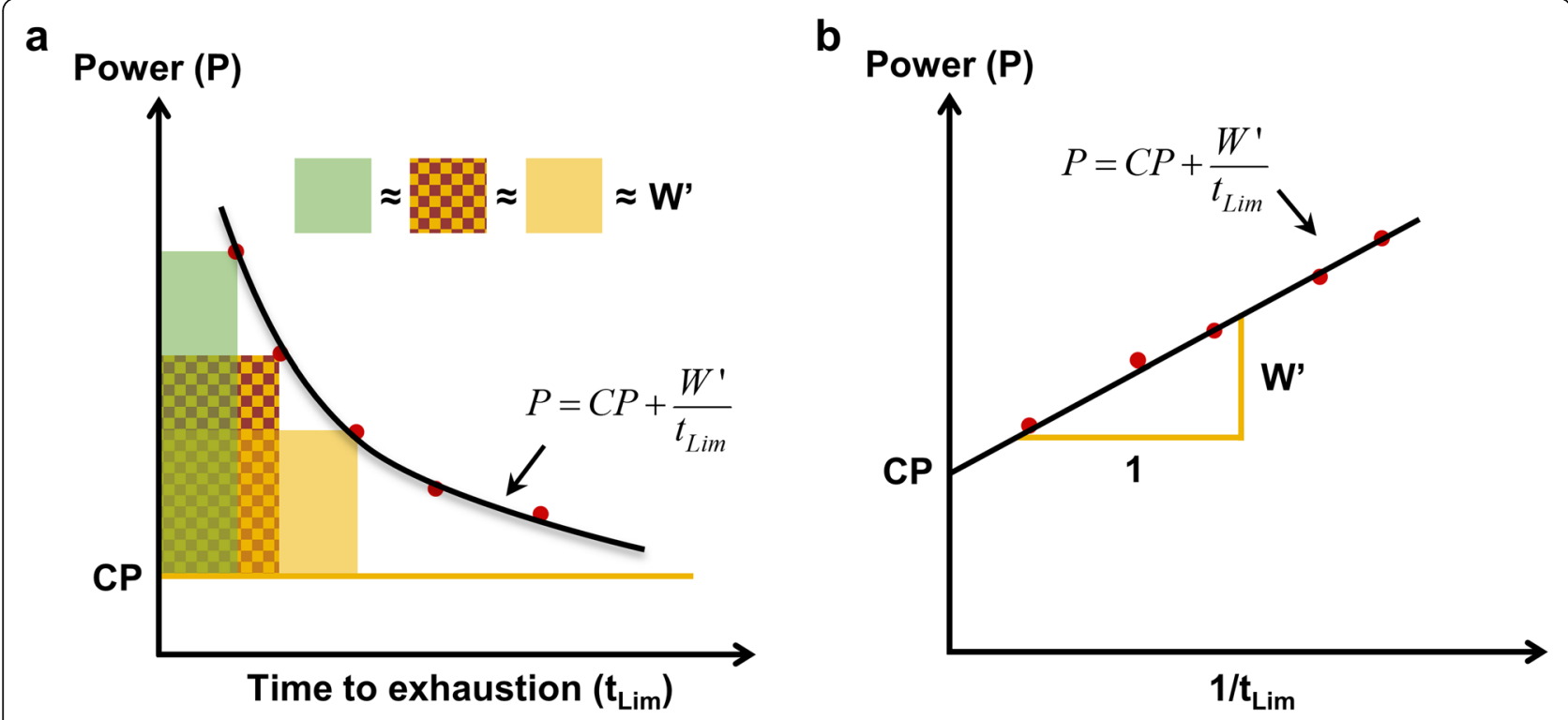

Fig. 1 The two-parameter model. a The hyperbolic form and $\mathbf{b}$ the linear transformation with critical power (CP) as the $\boldsymbol{y}$-intercept and curvature constant $\left(W^{\prime}\right)$ as the slope 
model was derived from the first law of thermodynamics incorporating both the anaerobic and aerobic contributions to the power generated given by:

$$
P(t)=\left(P_{\max }-R\right) \cdot e^{(-\lambda \cdot t)}+R
$$

where $P_{\max }$ is the maximum available power from the anaerobic mechanism, $R$ (analogous to $\mathrm{CP}$ ) is the maximum rate of energy release (power) from the aerobic mechanism, and $\lambda$ represents the decay of power with time $t$. Equation 6 is a simplified version of the model presented by Ward-Smith. The complete version can be found in [3]. Hopkins and colleagues [49] proposed a similar model for treadmill running with inclinations instead of power given by:

$$
I_{t}=I_{\infty}+\left(I_{0}-I_{\infty}\right) \cdot e^{(-t / \tau)}
$$

where $I_{\mathrm{t}}$ is the inclination at time $t, I_{\infty}$ is the inclination that corresponds to infinite time (similar to $\mathrm{CP}$ ), $I_{0}$ is the instantaneous maximum inclination (synonymous with $P_{\max }$ ), and $\tau$ is a time constant.

Peronnet and Thibault [50] built on Ward-Smith's work and proposed a model to predict race performances in the range of $60 \mathrm{~m}$ to a full marathon. Their main assumption was that the maximum aerobic power (analogous to CP) is sustainable for approximately $7 \mathrm{~min}$ as opposed to indefinitely as suggested by Eq. 5 . The model is given by:

$$
\begin{aligned}
& P_{T}=\frac{S}{T}\left[1-e^{\left(-T / k_{2}\right)}\right]+\frac{1}{T} \int_{0}^{T}\left[\mathrm{BMR}+B \cdot\left(1-e^{\left(-t / k_{1}\right)}\right)\right] d t \\
& \text { (i) } T<T_{\text {MAP }}\left\{\begin{array}{c}
S=A \\
B=\text { MAP-BMR }
\end{array}\right. \\
& \text { (ii) } T>T_{\mathrm{MAP}}\left\{\begin{array}{c}
S=A+A \cdot f \cdot \ln \left(T / T_{\mathrm{MAP}}\right) \\
B=(\mathrm{MAP}-\mathrm{BMR})+E \cdot \ln \left(T / T_{\mathrm{MAP}}\right)
\end{array}\right.
\end{aligned}
$$

where $P_{\mathrm{T}}$ is the power at any time $T, k_{1}$ and $k_{2}$ are respective time constants to account for the kinetics of aerobic and anaerobic metabolism, BMR is the basal metabolic rate assumed to be $1.2 \mathrm{~J} / \mathrm{kg}$ equivalent to 3.4 $\mathrm{ml} \mathrm{O}_{2} / \mathrm{kg} / \mathrm{min}$ using $1 \mathrm{ml} \mathrm{O}_{2}$ equivalent to $20.9 \mathrm{~J}, A$ is the capacity of the anaerobic metabolism in $\mathrm{J} / \mathrm{kg}, E$ is the reduction in peak aerobic power with natural logarithm of race duration $T$ (when $T>T_{\mathrm{MAP}}$ ), MAP is the maximum aerobic power in $\mathrm{W} / \mathrm{kg}, f$ is a constant describing reduction in energy from anaerobic metabolism over time $T$, and $T_{\mathrm{MAP}}$ is the time for which the MAP can be sustained (assumed to be $420 \mathrm{~s}$ ).

The Peronnet-Thibault model was able to estimate world record performances ranging from $60 \mathrm{~m}$ to full marathons with an average absolute error of $0.73 \%$ for males and $1.27 \%$ for females. The limitations, however, include the determination of the parameters $A$, MAP, and $E$ as well as the accuracy of the assumed parameters such as BMR and $T_{\text {MAP }}$ in Eq. 8. Morton [51] also discusses a bioenergetic hydraulic model with separate cases for maximal power, endurance at constant work rate, and endurance at incremental ramp exercises comprising of several parameters. Morton compares the bioenergetic model estimates to experimental data from other studies and shows them to be in agreement in predicting the endurance time for the different cases [51]. These alternate models, though having better accuracy in predicting the maximal instantaneous power and the maximal aerobic power compared to the two-parameter model, have many parameters that need to be assumed or estimated, which adds to their complexity [35, 52]. To address the limitations of the two-parameter model and to reduce the complexity of alternate models, Morton [52] proposed an extension of the two-parameter model by adding a non-zero time asymptote $k$ to Eq. 5 . This model was called the three-parameter model and is given by:

$$
t=\frac{W^{\prime}}{P-C P}+k
$$

where $\mathrm{k}$ can be derived by substituting $t=0$ and $P=$ $P_{\max }$ in Eq. 9 resulting in

$$
k=\frac{W^{\prime}}{C P-P_{\max }}
$$

Rewriting Eq. 9 as

$$
t=\frac{W^{\prime}}{P-C P}+\frac{W^{\prime}}{C P-P_{\max }}
$$

results in $k<0$ as $P_{\max }>\mathrm{CP}$. The $P_{\max }$ term in Eq. 11 represents the point at which the power curve intersects the power axis representing an instantaneous maximum power that can be produced.

Weyand and colleagues [53] proposed a model for allout cycling efforts ranging between 3 and $300 \mathrm{~s}$ which is similar to that of Ward-Smith's [3] given by:

$$
P(t)=P_{\text {aer }}+\left(P_{\text {mech } \max }-P_{\text {aer }}\right) \cdot e^{\left(-k_{\text {cycle }} \cdot t\right)}
$$

where $P_{\text {aer }}$ (synonymous with $\mathrm{CP}$ ) is the maximum power output that can be supported aerobically, $P_{\text {mech }}$ $\max$ is the maximum power output for a 3-s trial, and $k_{\text {cycle }}$ is the exponent describing the decrease in power with the increase in time $t$. Morton [54] also provided an extension of the three-parameter model while presenting a model for all-out running efforts given by:

$$
P(t)=C P+\left(P_{\max }-C P\right) \cdot e^{(t / k)}
$$

The model in Eq. 13 is similar to the models proposed by Ward-Smith [3], Hopkins and colleagues [49], and Weyand and colleagues [53]. Expressing Weyand's $k_{\text {cycle }}$ as a reciprocal will result in Hopkins' model in Eq. 7 and 
Morton's model in Eq. 13. The signs of these constants are different, which are accounted for by regression. Figure 3 shows three models (two-parameter, threeparameter, and exponential) plotted against experimental data presented by Gaesser and colleagues [34]. The values of $\mathrm{CP}, W^{\prime}$, and $P_{\max }$ were taken from [34], and data points were extracted using the open source software Plot Digitizer. Table 1 summarizes the estimates from each method.

There are other models proposed in the literature which are algebraic manipulations of the two-parameter model shown in Eq. 5. However, these models yield different estimates of $\mathrm{CP}$ and $W^{\prime}$ at the individual level for the same data as seen in $[34,55,56]$. These differences in estimates could originate from the rounding off approximations of reciprocals such as $1 / t_{\mathrm{Lim}}$. CP estimates from different models are reported to be in close agreement with each other in $[34,55,56]$. However, as illustrated in Table 1, the estimation of $W^{\prime}$ remains elusive as the same data can yield different estimates depending on the model used even though $\mathrm{CP}$ estimates are comparable [34, 38, 55-61]. The two-parameter model, though having limitations $(P=\infty$ at $t=0$ and CP lasting indefinitely), owing to its simplicity, can potentially be used to optimize performance as well as determining strategies by estimating time-to-exhaustion $[16,17,62]$.

\section{Methods and Protocols to Estimate CP and $W^{\prime}$}

The first protocol to estimate $\mathrm{CP}$ and $W^{\prime}$ was derived from Monod and Scherrer's [2] work. Subjects would complete at least three constant work-rate (CWR) to exhaustion tests, and the two-parameter model would then

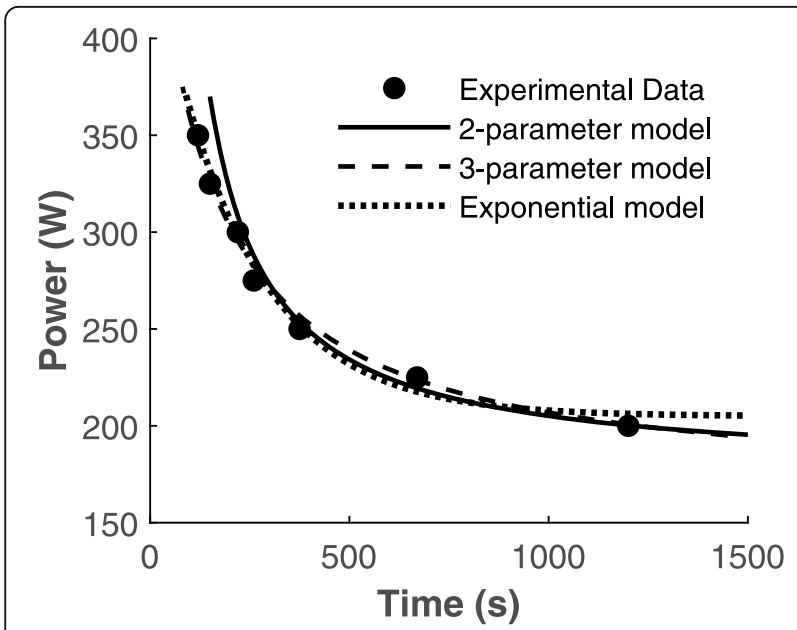

Fig. 3 The two-parameter model (solid line), the three-parameter model (dashed line), and the exponential model (dotted line) fitted to the same experimental data (solid circles) presented by Gaesser and colleagues [34]. Data extracted from Fig. 2 in [34] (p. 1434) and redrawn with permission using the values reported in the original article
Table 1 Summary of estimates from all models fit to the data presented by Gaesser and colleagues [34]

\begin{tabular}{|c|c|c|c|c|}
\hline Model & $\begin{array}{l}C P \\
(W)\end{array}$ & $W^{\prime}(J)$ & $\begin{array}{l}P_{\max } \\
(W)\end{array}$ & $\begin{array}{l}\text { Additional model parameters } \\
\left(\lambda, \tau, k_{\text {cycle, }} \text { or } k\right)(s)\end{array}$ \\
\hline $\begin{array}{l}\text { Two-parameter } \\
\text { model }\end{array}$ & $\begin{array}{l}1 \\
176\end{array}$ & $\begin{array}{l}2 \\
29100\end{array}$ & $3 \mathrm{NA}$ & $4 \mathrm{NA}$ \\
\hline $\begin{array}{l}5 \text { Three- } \\
\text { parameter model }\end{array}$ & $\begin{array}{l}6 \\
165\end{array}$ & $\begin{array}{l}7 \\
47900\end{array}$ & $\begin{array}{l}8 \\
491\end{array}$ & $9-146.93$ \\
\hline $\begin{array}{l}10 \text { Exponential } \\
\text { model }\end{array}$ & $\begin{array}{l}11 \\
205\end{array}$ & $\begin{array}{l}12 \\
\text { NA }\end{array}$ & $\begin{array}{l}13 \\
452\end{array}$ & 140.0044 or $-225.2867^{*}$ \\
\hline
\end{tabular}

*Morton's [54] $k=-225.2867$, Hopkins' [49] $\tau=225.2867$, which are same as Weyand's [53] $-1 / k_{\text {cycle }}$ and Ward-Smith's [3] $-1 / \lambda$

be fit to the data resulting in $\mathrm{CP}$ and $W^{\prime}$ estimates. D. W. Hill [35] suggests the use of the linear model ( $P$ versus $\left.1 / t_{\text {Lim }}\right)$ with at least $4-5$ CWR tests to arrive at CP and $W^{\prime}$ estimates.

While less prevalent in the literature, Morton [58] demonstrated another method to determine estimates of $\mathrm{CP}$ and $W^{\prime}$ from ramp exercises to exhaustion by deriving an equation between time-to-exhaustion and ramp slope given by:

$$
T=\frac{C P}{S}+\sqrt{\frac{2 \cdot W^{\prime}}{S}}
$$

where $T$ is the time-to-exhaustion in seconds and $S$ is the ramp slope in watts/second. Morton suggested that subjects complete $4-5$ ramp tests to exhaustion at different slopes. The time-to-exhaustion from these tests are then plotted against the slopes and Eq. 14 would be fitted to the data to determine CP and $W^{\prime}$. Morton claims that the estimates from this protocol appear to be lower than those from the CWR protocol thus addressing the overestimation of $\mathrm{CP}$ reported in a few publications cited earlier. The ramp protocol was compared to the CWR protocol by Morton and colleagues [63] showing an underestimation of $W^{\prime}$ and no statistical difference for CP. However, a closer inspection shows underestimation of $W^{\prime}$ by approximately $10 \mathrm{~kJ}, 4 \mathrm{~kJ}, 3 \mathrm{~kJ}$, and $9 \mathrm{~kJ}$ for subjects 1,2, 3, and 6 respectively and an overestimation of $W^{\prime}$ by approximately $8 \mathrm{~kJ}, 6 \mathrm{~kJ}$, and $3 \mathrm{~kJ}$ for subjects 4 , 9 , and 10 respectively (see Table 1 in [63]).

Vanhatalo and colleagues [64] proposed the 3-min allout test (3MT) to determine $\mathrm{CP}$ and $W^{\prime}$ in fewer laboratory visits. This test involves pedaling at all-out intensity for $3 \mathrm{~min}$ with $\mathrm{CP}$ estimated by the average power from the last $30 \mathrm{~s}$ and $W^{\prime}$ given by the area under the curve above CP [57, 64]. Figure 4 shows the schematic representation of a notional 3MT. Parallels can be drawn between the 3MT and the Wingate anaerobic test [65], which is essentially a 30 -s all-out test. Studies that compare $W^{\prime}$ to the anaerobic capacity from the Wingate test report a correlation coefficient of $\sim 0.7[66,67]$. 


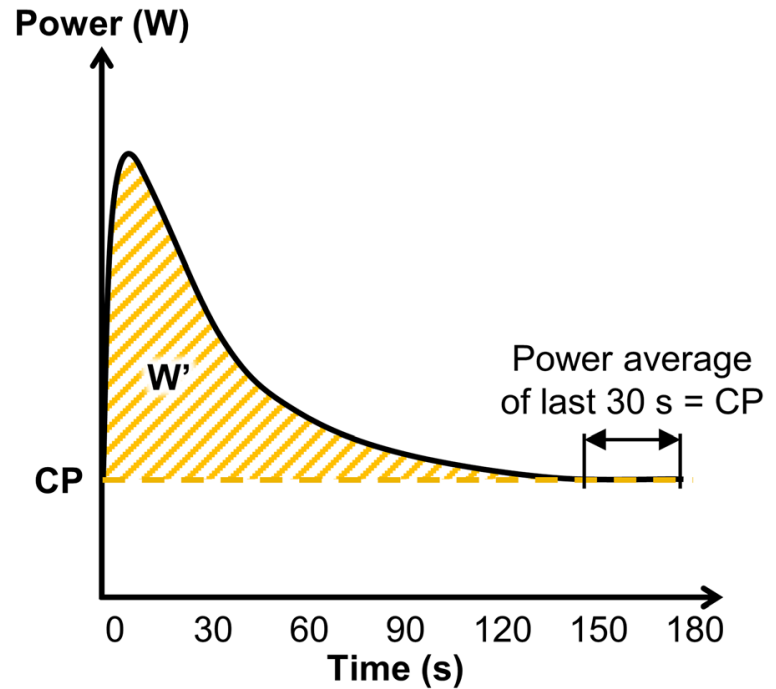

Fig. 4 Schematic representation of a 3-min all-out test to determine critical power $(C P)$ and the curvature constant $\left(W^{\prime}\right)$. The average power of the last 30s yields CP and the area below the curve and above CP yields $W^{\prime}$

Therefore, the anaerobic capacity from the Wingate test and $W^{\prime}$ cannot be used interchangeably.

The estimates from the $3 \mathrm{MT}$ have been compared to those from the CWR tests in $[28,60,68]$ and thereby, validating the $3 \mathrm{MT}$. Burnley and colleagues [69] saw (in 7 out of 11 subjects) a steady state blood lactate and oxygen uptake profile in $30 \mathrm{~min}$ of exercise at $15 \mathrm{~W}$ below $\mathrm{CP}$ determined from the $3 \mathrm{MT}$. They made the same subjects pedal at $15 \mathrm{~W}$ above CP which resulted in an average time-to-exhaustion of $13 \pm 7 \mathrm{~min}$. Black and colleagues [62] used the CP determined from the $3 \mathrm{MT}$ to successfully estimate a $16.1 \mathrm{~km}$ time trial performance. However, studies have reported that the time-to-exhaustion at $\mathrm{CP}$ derived from the $3 \mathrm{MT}$ to be $14.79 \pm 8.38 \mathrm{~min}$ and $12.5 \pm 6.5$ $\min$ in $[70,71]$ respectively. These are similar to $13 \pm$ $7 \mathrm{~min}$ for exercise at $15 \mathrm{~W}$ above $\mathrm{CP}$ reported by Burnley and colleagues [69]. Moreover, $W^{\prime}$ from $3 \mathrm{MT}$ has also been reported to be overestimated in comparison to CWR protocol $(11.37 \pm 3.84 \mathrm{~kJ}$ vs $9.55 \pm 4$ kJ) [72]. However, as discussed by Skiba [73], the errors observed in the estimates could be attributed to not using the same equipment or not adhering to the test procedure laid out in [64]. Additionally, the inherent day-to-day variability within subjects, referred to as the intra-individual variability (IIV), may have contributed to the shorter time-to-exhaustion observed at CP $[17,38]$. Hence, exercise outside a subject's 95\% confidence interval of $\mathrm{CP}$, i.e., outside the bounds of the IIV associated with CP (similar to 15 W above and below CP in [69]), will yield better insights into reliability of the $3 \mathrm{MT}$.
Limitations of the Protocols Used to Determine CP and $W^{\prime}$ The CWR protocol is considered as the "gold-standard" to estimate CP and $W^{\prime}$ as it was the first method to be proposed. However, the CWR protocol is not devoid of shortcomings. Using the CWR protocol, Bishop and colleagues [74] and Jenkins and colleagues [75] illustrated that the duration of the predicting trials influences the estimates with both $\mathrm{CP}$ and $W^{\prime}$ computed from three shortest duration trials being significantly greater than those from the three longest trials. Furthermore, CP estimates from the CWR protocol at $60 \mathrm{rpm}$ have been found to be significantly greater than those at $100 \mathrm{rpm}$ [76]. Considering these limitations, Muniz-Pumares and colleagues [61] suggest the use of the two-parameter hyperbolic model with at least three CWR trials of durations $>2 \mathrm{~min}$ and $<15 \mathrm{~min}$ and freely chosen cadence to arrive at reliable estimates.

The 3MT avoids the need to do multiple tests to arrive at $\mathrm{CP}$ and $W^{\prime}$. However, there are reports of overestimation of $\mathrm{CP}$ from the $3 \mathrm{MT}[70,71,77]$, which are comparable to other reports of overestimation of CP from the CWR tests in [41-44]. The $3 \mathrm{MT}$ appears to reliably predict a $16.1 \mathrm{~km}$ time trial performance [62], which is in accordance with other studies that report the validity of $\mathrm{CP}$ to be $40 \mathrm{~min}$ to over $1 \mathrm{~h}[35,45,46]$. These contradictory results can be attributed to equipment, test method, validation methods, and the day to day variability of the participants [17, 38, 73].

It has been shown that the day-to-day (or trial-totrial) variability within a person, i.e., IIV, affects performance during physical activities in [78]. The CWR tests, depending on the fit and the model used, yield standard errors of estimation (SEEs) for CP and $W^{\prime}$. These SEEs give a measure of goodness of fit and not the IIV. To truly capture and quantify IIV using the CWR protocol, exercise to exhaustion at each workrate must be repeated multiple times. $\mathrm{CP}$ and $W^{\prime}$ estimates for each set of tests could be determined, which can then be averaged to arrive at a grand mean for $\mathrm{CP}$ and $W^{\prime}$ (see Fig. 5). On similar lines, Triska and colleagues [79] conducted maximal effort time trials spanning 3,7 , and $12 \mathrm{~min}$ with each trial repeated thrice (one familiarization and two repeats) and computed $\mathrm{CP}$ and $W^{\prime}$ for each data set using the two-parameter hyperbolic model. They found higher reliability between the post familiarization trials with intra-class correlation coefficient of 0.95 and 0.94 and a coefficient of variation of $2.6 \%$ and $8.2 \%$ for $\mathrm{CP}$ and $W^{\prime}$ respectively. However, an average $\mathrm{CP}$ and $W^{\prime}$ for all three sets of data (or post familiarization trials) could be computed to yield grand means for CP and $W^{\prime}$ for each subject with their IIVs as shown in Fig. 5. Although costly in terms of time, this method may 


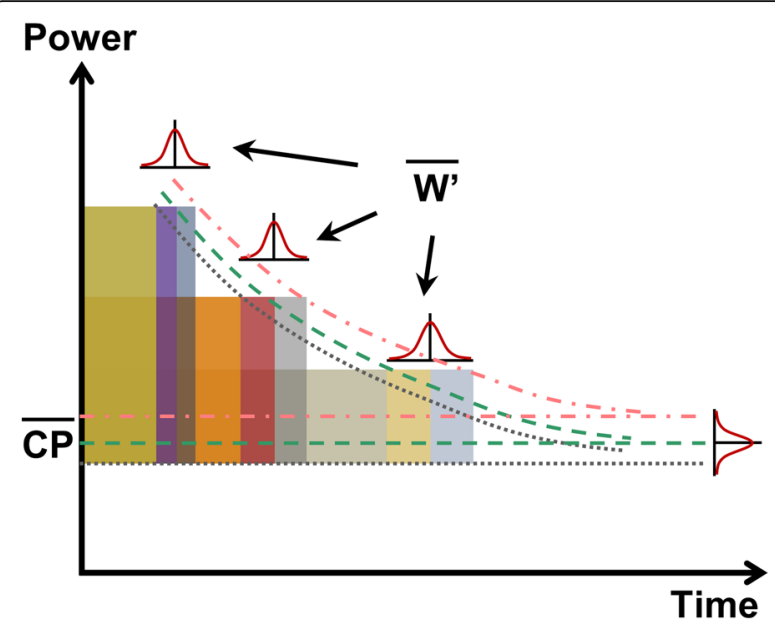

Fig. 5 Repeated constant work-rate (CWR) tests to capture intraindividual variability (IIV) associated with critical power (CP) and curvature constant $\left(W^{\prime}\right)$ estimates. The dotted, dashed, and dotdashed lines show the fits to the different sets of data and their respective asymptotes. The grand means for $\mathrm{CP}$ and $W^{\prime}$ are obtained by averaging the respective parameters estimates from each curve fitting

lead to a better understanding of $W^{\prime}$, which has been shown to be ambiguous and significantly dependent on the mathematical model used [34, 55-57, 59-61].

Though the 3MT has been shown to be repeatable in [69], a closer investigation of the Bland-Altman plots presented in the first paper on 3MT [69] (p.1998, Fig. 1d) shows the bias and $95 \%$ limits of agreement of $-1 \pm$ $15 \mathrm{~W}$ resulting from the variability associated with each subject's CP estimate across two trials. A 15-W change in CP between two 3MTs contributes to a difference of $2700 \mathrm{~J}$ of $W^{\prime}$ across the 3 min of the test. This IIV needs to be accounted for before prescribing training schedules and interventions based on the 3MT. The estimates from the CWR protocol have associated SEEs for CP and $W^{\prime}$, whereas it is not possible to get a standard error for $W^{\prime}$ from a 3MT. A possible way to arrive at SEEs for $\mathrm{CP}$ and $W^{\prime}$ from the $3 \mathrm{MT}$ is by fitting a curve to the data. Morton [54] used a biexponential extension to his exponential model (Eq. 13) to be applicable to all-out efforts given by,

$$
P(t)=C P+\left(P_{\max }-C P\right) \cdot e^{t / k}+P_{\mathrm{IN}} \cdot e^{-t / k^{\prime}}
$$

where $P$ is the power at any time $t, \mathrm{CP}$ is the critical power, $P_{\max }$ is the instantaneous maximum power, $P_{\mathrm{IN}}$ is the power required to overcome the initial inertial resistance of the ergometer flywheel, and $k$ and $k^{\prime}$ are constants. The $P_{\mathrm{IN}}$ term accounts for $0--5 \mathrm{~s}$ of the all-out test. The model in Eq. 15 is shown to fit the all-out test data with $R^{2}=0.985$ in [54]. However, it has the following shortcomings:
- At $t=0, P(0)=P_{\max }+P_{\mathrm{IN}}$, which is not possible as the instantaneous maximum power that can be generated is $P_{\text {max }}$. Instead, at $t=0, P(0)=P_{\max }-$ $P_{\mathrm{IN}}$ is a more realistic power output. The $P_{\max }-P_{\mathrm{IN}}$ correction is a mathematical quirk and lacks physiological basis. However, $P_{\max }$ could be assumed to be equal to either the average power output of one crank-rotation [40] or the power output of 3-s trial [53] which accounts for the physiological constraints of producing an instantaneous $P_{\max }$. Furthermore, if the all-out interval starts from rest, then at $t=0, P(0)=0$ is a more valid initial condition as power is defined as energy-expended/time and no energy is expended before starting the exercise.

- Morton fit the model to Burnley's data in [69] which resulted in the $\mathrm{CP}=336.3 \pm 1.2 \mathrm{~W}, P_{\max }=959.3 \pm$ $7.9 \mathrm{~W}, P_{\mathrm{IN}}=512.1 \pm 13.8 \mathrm{~W}, k=-29.9 \pm 0.5 \mathrm{~s}$, and $k^{\prime}=3.14 \pm 0.16 \mathrm{~s}$. Using these values in Eq. 15 and plotting against time (from 0 to $180 \mathrm{~s}$ ) does not result in the desired shape of the $3 \mathrm{MT}$ as shown in Fig. 4 (see Fig. 6). If $P_{\text {IN }}$ were to be negative, the resulting shape would be similar to that of Fig. 4. However, a negative resistance for the flywheel is unrealistic.

- The power required to overcome the inertial resistance of the flywheel can be computed using Newton's second law for rotational motion as shown in [80]. The $P_{\text {IN }}$ term is a function of torque and acceleration. Thus, there is no reason to assume an exponential decay as shown in Eq. 15. A piecewise model could be developed for a 3MT with the first piece to account for the power needed to overcome the flywheel's inertia and the second to account for the decline from peak power to CP. Furthermore,

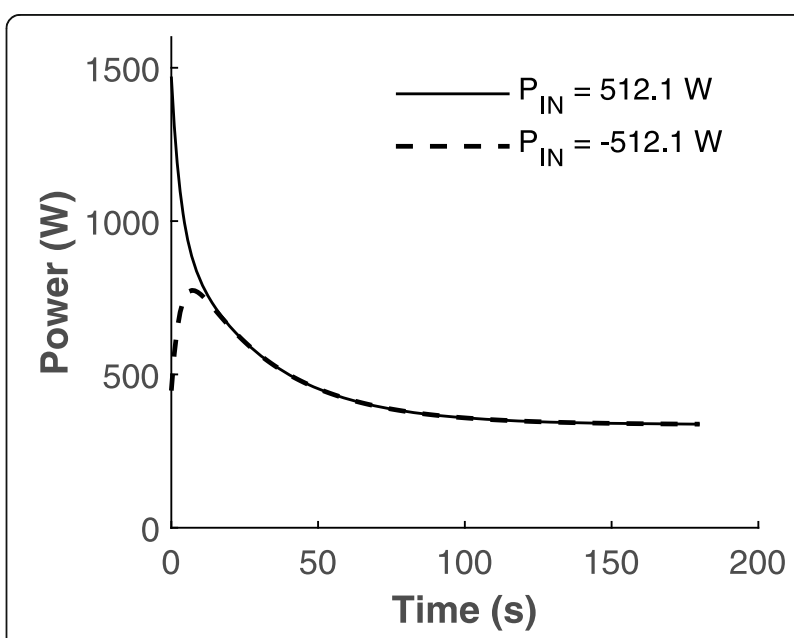

Fig. 6 Morton's biexponential model [54] plots showing positive inertial resistance of ergometer flywheel, $P_{\mathbb{I N}}$ (solid line) and negative $P_{\mathbb{I N}}$ (dashed line). The positive $P_{\mathbb{I N}}$ term does not yield the shape shown in Fig. 4 
the time taken by the muscle to reach $P_{\max }$ needs to be accounted for in the first piece where the muscles are overcoming the flywheel resistance while reaching their maximal power output.

The SEEs from curve fitting, as mentioned earlier, do not quantify the IIV associated with $\mathrm{CP}$ and $W^{\prime}$ for an individual. Conducting multiple tests and computing grand means for $\mathrm{CP}$ and $W^{\prime}$ from each set of tests significantly increases the time investment. There is a need for better methods to capture the IIV from a 3MT, minimize the number of testing days, and statistically compare two 3MTs to arrive at reliable estimates of $\mathrm{CP}$ and $W^{\prime}$ for an individual. Furthermore, most studies report the average of their participant groups. While this is convenient in terms of comparing them with estimates from other methods and protocols, they give little information pertaining to the repeatability and variability at the individual level. It is, therefore, practical to consider individuals rather than groups and arrive at athletespecific models. This is important in terms of modeling recovery of $W^{\prime}$ which could be appended to the twoparameter $\mathrm{CP}$ model, thereby aiding in performance optimization.

\section{Adding Recovery to the Two-Parameter Model}

The CP concept has been discussed using a hydraulic vessel analogy by Morton [38]. Morton [38] discusses that the aerobic and anaerobic domains are analogous to energy vessels connected by a tube of fixed diameter, with the anaerobic vessel being limited in capacity and the aerobic being unlimited (see Fig. 7). Morton suggests that when functioning above $\mathrm{CP}$, energy is derived from

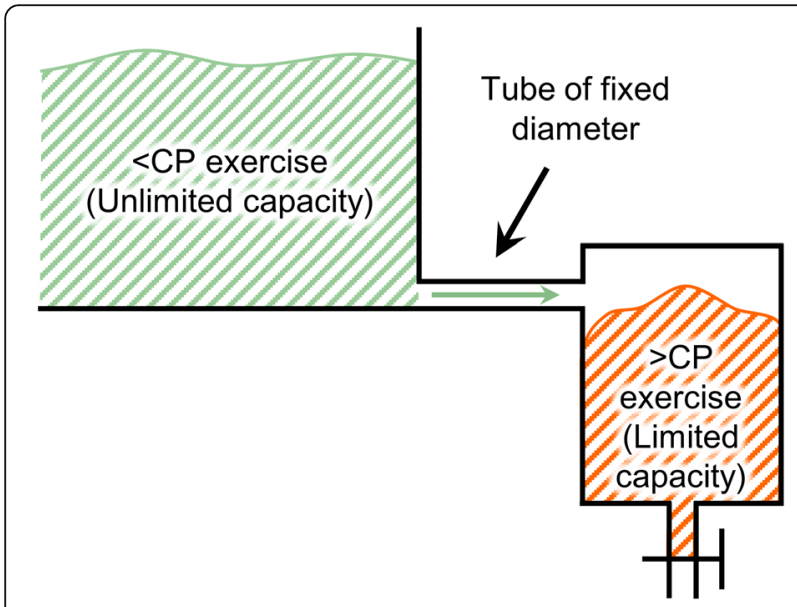

Fig. 7 Critical power (CP) concept using Morton's hydraulic vessel analogy [38]: Energy domains show sub-CP and supra-CP vessels connected by a tube of fixed diameter. Morton's aerobic and anaerobic vessels are replaced by $\angle C P$ and $>C P$ respectively as the curvature constant $\left(W^{\prime}\right)$ and anaerobic work capacity (AWC) cannot be used interchangeably the anaerobic vessel, whereas when exercising below CP, energy is supplied by the aerobic vessel. Morton's hydraulic analogy considers $\mathrm{CP}$ to be the boundary between aerobic and anaerobic domains, and AWC to be equal to $W^{\prime}$ as it was published around the same time as Dekerle and colleagues' study [33] that showed that AWC and $W^{\prime}$ cannot be used interchangeably.

Ignoring the assumption of $\mathrm{AWC}$ and $W^{\prime}$ being equal, Morton's analogy suggests that while below $\mathrm{CP}$, the curvature constant $W^{\prime}$ (limited capacity tank in Fig. 7) is refilled or recovered. This suggestion presents the possibility of modeling the recovery of $W^{\prime}$ while exercising below $\mathrm{CP}$ and thereby, together with the two-parameter model, optimizing performance. While there are models to estimate the depletion of $W^{\prime}$, there are only a few models that attempt to estimate its recharge/recovery while below $\mathrm{CP}$.

The first model considering recovery of $W^{\prime}$ was proposed by Morton and Billat [81]. Morton and Billat [81], based on the two-parameter model, derived an equation for time-to-exhaustion for intermittent exercise by assuming that the rates of recharge and expenditure of $W^{\prime}$ were equal given by:

$$
t=\frac{n \cdot\left(t_{w}+t_{r}\right)+W^{\prime}-n \cdot\left[\left(P_{w}-C P\right) \cdot t_{w}-\left(C P-P_{r}\right) \cdot t_{r}\right]}{\left(P_{w}-C P\right)}
$$

where $t$ is the total duration of the intermittent exercise, $n$ is the number of intervals, $t_{\mathrm{w}}$ and $t_{\mathrm{r}}$ are respective durations of intervals above and below $\mathrm{CP}$, and $P_{\mathrm{w}}$ and $P_{\mathrm{r}}$ are respective power outputs of intervals above and below CP. Ferguson and colleagues [82] were first to quantify recovery of $W^{\prime}$ by proposing that it is "curvilinear" and not proportional to its depletion as assumed by Morton and Billat [81]. Acknowledging this curvilinear nature of recovery of $W^{\prime}$, Skiba and colleagues [83-86] proposed a model which assumed the behavior to be exponential given by:

$$
W_{b a l}^{\prime}=W^{\prime}-\int_{0}^{t} W_{\text {exp }}^{\prime} \cdot e^{-\left(\frac{(t-u)}{\tau} W^{\prime}\right)} d u
$$

where $W^{\prime}$ bal is the $W^{\prime}$ balance at any time during exercise, $W^{\prime} \exp$ is the amount of $W^{\prime}$ expended, $(t-\mathrm{u})$ is the duration of the recovery interval, and $\tau_{W}$ is the time constant of reconstitution of $W^{\prime}$ in seconds given by:

$$
\tau_{W^{\prime}}=546 \cdot e^{\left(-0.01 D_{C P}\right)}+316
$$

where $D_{\mathrm{CP}}$ is the difference between $\mathrm{CP}$ and average power output during all intervals below CP. Eq. 18 is a non-linear regression obtained by plotting $\tau_{W^{\prime}}$ values 
(calculated by setting the $W^{\prime}$ bal $=0$ in Eq. 17 at the termination of exercise) against respective $D_{\mathrm{CPs}}$.

Skiba's model was validated in [84] where an average $W^{\prime}$ balance at exhaustion of $0.5 \pm 1.3 \mathrm{~kJ}$ was reported. However, the model cannot be used to determine $W^{\prime}$ balance in real time [73] (p.78) as the $\tau_{W^{\prime}}$ term needs $W$ 'bal to be zero which is not known until the termination of each test. Moreover, three forms of the $W^{\prime}$ bal model have been published by Skiba and colleagues [83-86]. The first [83] contains only the integrand and not the differential variable. The second $[84,85]$ contains the differential "du" as shown in Eq. 17, whereas the third [86] has "dt" as its differential variable. Changing the differential variable from "du" to "dt" yields different results upon integration. Additionally, inspecting Eq. 17 reveals that the integral term on the right-hand side has units of Joules-second causing an inequality as the units of the left-hand side are Joules. Additional file 1 of this manuscript provides a detailed derivation of the mathematical solutions for both "du" and "dt" as the differential term of the $W^{\prime}$ bal model illustrating the difference in results as well as the imbalance of units. Furthermore, the standard errors associated with the estimation of CP and $W^{\prime}$ may cause a negative balance of $W^{\prime}$ balance (can be seen in [84], Fig. 2, p.903). Skiba and colleagues [85] proposed a biconditional $W^{\prime}$ bal model which resolves the inequality of units (can be seen in Appendix 1 of [85]) given by:

$$
\begin{aligned}
& \text { If } P>C P, \quad W^{\prime} \text { bal }=W^{\prime}{ }_{0}-[(P-C P) \cdot t] \\
& \text { If } P<C P, \quad W^{\prime}{ }_{\text {bal }}=W^{\prime}{ }_{0}-W^{\prime}{ }_{\text {exp }} \cdot e^{\left(\frac{-D_{C P} \cdot t}{W_{0}^{\prime}}\right)}
\end{aligned}
$$

where $W^{\prime}{ }_{0}$ is $W^{\prime}$ at time $t=0$. Though the model in Eq. 19 resolves the inequality of units, it has been shown to underestimate the recovery of $W^{\prime}$ by Bartram and colleagues [87]. Bickford and colleagues [88] presented a model of recovery of $W^{\prime}$ which was derived from limited data and thus needs refinement.

Apart from the models presented above, at the time of submission, there are no models available in the literature that attempt to model the recovery of $W^{\prime}$. These models need to be improved for accurately modeling the recovery of $W^{\prime}$ and combining them with the models of exertion that are well established in the literature. There is potential in extending the two-parameter model to include the recovery model. A combined exertionrecovery/discharge-recharge model of $W^{\prime}$ will be worthwhile in estimating the time-to-exhaustion of endurance efforts and optimizing performance. The potential of optimizing performance to accomplish a 2-h marathon has been illustrated by Nike's Breaking2 project [89] which has inspired modeling studies by Hoogkamer and colleagues [90-92] based on the two-parameter CP model with exponential recovery similar to Eq. 19, biomechanical improvements, shoe design improvements, and drafting strategy. Furthermore, the successful completion of a sub 2-h marathon by Eliud Kipchoge as a part of the INEOS 1:59 challenge in Vienna in October 2019 provides encouraging signs for investigative studies focusing on optimization of performance in other endurance sports.

\section{Applications of a Combined Expenditure-Recovery Model of $W^{\prime}$}

In the literature reviewed thus far, studies modeling recovery of $W^{\prime}$ are scarce. A few models attempt to address the need for a combined expenditure-recovery model. Skiba's first model [83] is similar to the monoexponential ventilatory gas exchange model for moderate intensity cycling proposed by Whipp and colleagues [93] and Vandewalle and colleagues' aerobic power model [67]. The exponential assumption of recovery seems logical as sub-CP exercise is considered to be supported by aerobic mechanisms [38]. The $\tau_{W^{\prime}}$ relation in Eq. 18 is representative of the seven recreational athletes from whose data it was derived. Though the model was validated using data from eight triathletes [84], it may not be able to predict the recovery of $W^{\prime}$ for athletes of higher or lower caliber. This is illustrated by Caen and colleagues [94] where faster recovery of $W^{\prime}$ was observed. Skiba's second model (also mono-exponential) [85], derived from first principles with valid assumptions, addresses some limitations of the earlier version. However, it has not been validated and, like its predecessor, has been shown to have slower recovery kinetics for elite athletes by Bartram and colleagues [87].

De Jong and colleagues [95] have used the twoparameter model to simulate the optimization of a $5-\mathrm{km}$ time-trial performance. However, a recovery model in combination with the two-parameter model will aid in optimizing performance over longer durations and distances. There have been other attempts at combining the two-parameter model with a recovery model [88], but the limited data result in the need for refinement. The advantage of an exertion-recovery model is the ability to accurately predict the time-to-exhaustion during endurance exercises. Furthermore, modeling fatigue, exhaustion, and recovery has applications not only in the field of athletic training and performance but also in the fields of medicine and health monitoring $[12,16,17]$.

With an exertion-recovery model based on the CP concept, an energy management system can be designed that will regulate the expenditure and recovery of $W^{\prime}$. The optimization objectives would be minimizing time and maximizing distance by maximizing power output with the help of an exertion-recovery model. For example, in cycling races, 3-4 cyclists form pelotons to reduce drag. It has been 
shown that the cyclists in the middle of a peloton experience up to $40 \%$ less drag [96]. A potentially successful race strategy for the peloton group can be derived from the exertion-recovery model using CPs and $W^{\prime}$ s of the individual riders. A similar drafting strategy was employed by Eliud Kipchoge in the INEOS 1:59 challenge where he completed a full marathon in $1 \mathrm{~h} 59 \mathrm{~min}$ and $40.2 \mathrm{~s}$. Another application is an energy management system for foot missions of soldiers. Time to exhaustion in long foot missions, where soldiers carry all the load of ammunition, food, and water can be accurately estimated with an exertionrecovery model. Additionally, in team sports like football, rowing, lacrosse, and soccer, $\mathrm{CP}$ and $W^{\prime}$ could be used in team selection, determining team strategies, planning individual training needs, and training interventions [97]. Furthermore, the combined model can be used to link $W^{\prime}$ balance to performance quality and to estimate injury risk. Together with wearable sensors, the model could potentially be used to determine team strategies in terms of player substitutions and avoiding fatigue-related injuries and for real-time performance optimization. The rise in popularity of wearable sensors has resulted in their use in health monitoring [98] and physical activity tracking [98, 99] and provides opportunities to mitigate dependence on laboratory equipment. Therefore, models of human performance can be tested and validated outside the laboratory.

Research Opportunities in Modeling Human Performance The research opportunities identified in this review article are cross-functional encompassing the areas of human performance, exercise physiology, health, and engineering. Though the themes belong to different backgrounds, they are not independent of each other. Table 2 summarizes the theme-wise research opportunities and applications that have been identified in this paper.

Developing mathematical models of fatigue will not only aid athletes, but also defense personnel in mission planning and healthcare professionals who study the effect of physical exertion on overall health. The ability to quantify the day-to-day variability aids the measurement of training effectiveness and training prescription. Furthermore, the theory of expenditure of $W^{\prime}$ is explained well by the two-parameter model. However, a robust model for recovery of $W^{\prime}$ is yet to be proposed.

\section{Conclusions}

The objective of this paper was to review the state of the art for power-based models of fatigue and identify opportunities to advance the field. Power based models of human performance which have their origins in cycling have been reviewed. The two-parameter $\mathrm{CP}$ concept reliably estimates fatigue due to severe intensity exercise in the range of $2 \mathrm{~min}$ to $1 \mathrm{~h}$ and is also suitable to model sprint performances of appropriate durations. Alternate models predict the power and time relationship in the severe intensity domain with better accuracy, but these models require the determination of more parameters, thereby, increasing complexity. $\mathrm{CP}$ and $W^{\prime}$ can be estimated using multiple models and protocols with the 3MT being the least time-consuming method. The 3MT,

Table 2 Theme-wise research opportunities and applications of human performance modeling

\begin{tabular}{ll}
\hline Themes & Research opportunities and applications \\
\hline Groups versus individuals & Models derived from the data pertaining to a group of individuals may not accurately \\
& model performance of athletes outside the group, thus, suggesting a need for individual \\
& specific models [87]. \\
& Understanding of $W$ is still ambiguous as it is dependent on the model used \\
Influence of mathematical modeling on $W^{\prime}$ & {$[34,55-57,59-61]$. Quantifying the natural day-to-day/trial-to-trial variability within subjects, } \\
& i.e., IIV, may yield a better understanding of $W$.
\end{tabular}

Natural variability within an individual

Methods need to be developed to quantify the IIV associated with physiological parameters, which will be useful in measuring training effectiveness, developing higher fidelity models, and optimizing performance.

Recovery of $W^{\prime}$

Current models described in [83-88] need refinement and improvement. A robust model for recovery of $W^{\prime}$ is needed, which could be athlete-specific. The $W^{\prime}$ balance can potentially be correlated to fatigue related injuries and the risk of injury could be estimated.

Performance optimization

The recovery model in conjunction with the two-parameter model enables optimization of time-trial performance as illustrated in $[95,100]$ and illustrated in $[91,92]$.

Wearable sensor integration

Wearable sensors provide opportunities in real-time performance tracking, optimization, and methods to reduce the reliance on laboratory equipment. Similar to studies in [101, 102], commercially available sensors could be validated against laboratory equipment and used in the field for developing higher fidelity models.

Integration of individual performance modeling into team performance

Physical exertion and health
Athlete-specific models could be used in determining team strategies, training interventions, planning training needs, and team selection as illustrated in [91, 97].

Models of human performance could be used to gain insight into the effect of physical exertion on overall health and well-being as discussed in $[16,17]$. 
despite its advantages, has a limitation of not capturing the IIV associated with $\mathrm{CP}$ and $W^{\prime}$ estimates. Standard errors associated with the estimates from the powertime regression of CWR tests could help in better quantifying this variability. However, they only give a measure of goodness of fit and do not capture the IIV. None of the models available accommodate the IIV associated with the parameter estimates, regardless of the method of estimation used. Until methods to capture IIV are proposed and validated, subject-specific training prescription and subsequent performance optimization will be limited in precision and accuracy. Additionally, models derived from group data do not represent the population as several factors and variables have a bearing on human performance. Individualized athlete-specific models need to be derived to potentially improve performance through training prescriptions. The $\mathrm{CP}$ concept, owing to its simplicity, is promising and robust in terms of modeling fatigue in the severe intensity domain. However, it is incomplete due to the lack of proper understanding of the recovery behavior of $W^{\prime}$ in the moderate and heavy intensity domains. Attempts have been made to address this gap, but with limited success. The models available provide a good starting point to develop models of higher accuracy and fewer assumed parameters. A combined exertion-recovery model will lead to optimized performance realized through an energy management control system. The combined model could lead to a straightforward way of assessing fatigue and risk of injury and have implications with respect to the influence of exercise on overall health.

\section{Supplementary information}

Supplementary information accompanies this paper at https://doi.org/10. 1186/s40798-019-0230-z.

Additional file 1. Derivation of mathematical solutions for the different forms of the $W_{\text {bal }}$ model presented by Skiba and colleagues.

\begin{abstract}
Abbreviations
3MT: 3-min all out test; AWC: Anaerobic work capacity; BMR: Basal metabolic rate; CP: Critical power; CV: Critical velocity; CWR: Constant work-rate; D ': Curvature constant of velocity-time relationship; $D_{\mathrm{Cp}}$ : Difference between critical power and the average recovery power; GET: Gas exchange threshold; IIV: Intra-individual variability; LT: Lactate threshold; MAP: Maximum aerobic power; MLSS: Maximal lactate steady state; $P_{\text {aer: }}$ Maximum power output supported aerobically; $P_{\mathbb{I N}}$ : Power to overcome the inertia of the flywheel of the ergometer; $P_{\max }$ : Instantaneous maximum power; $P_{\text {mech max }}$ : Maximum power output for a $3 \mathrm{~s}$ trial; SEE: Standard error of estimation; $t_{\text {Lim: }}$ Time to exhaustion; $T_{\text {MAP: }}$ Time to exhaustion at maximum aerobic power; $V_{\text {max: }}$ : Maximum muscle shortening velocity; $\dot{V}_{2}$ : Oxygen uptake; $\mathrm{V}_{2 \text { max }}$ : Maximal oxygen uptake; $W$ : Curvature constant of the power-time relationship; $W_{\text {bal: }}^{\prime} W^{\prime}$ balance; $W_{\text {Lim: }}$ Limit work
\end{abstract}

Acknowledgements

Not applicable.

\section{Authors' Contributions}

VSMS, GMM, and REH performed the literature search, compiled the results and wrote the paper. VSMS contributed to the literature search, compiled the results, wrote, formatted, and edited the original draft, and created the visualizations. G.M.M and R.E.H contributed in writing, reviewing, and performing the final edits of the manuscript. All authors read and approved the final manuscript.

\section{Funding}

No funding was received to assist the preparation of this article.

\section{Availability of Data and Materials}

Not applicable as data were neither generated nor analyzed in the current study. The supplementary material contains detailed mathematical solutions of the different forms of the $W_{\text {bal }}$ model.

Ethics Approval and Consent to Participate

Not applicable

\section{Consent for Publication}

Not applicable

\section{Competing Interests}

The authors, Vijay Sarthy M Sreedhara, Gregory M Mocko, and Randolph E Hutchison, declare that they have no competing interests.

\section{Author details}

${ }^{1}$ Department of Mechanical Engineering, Clemson University, 243 Fluor Daniel EIB, Clemson, SC 29634-0921, USA. ²Department of Health Sciences, Furman University, Greenville, SC 29613, USA.

Received: 20 August 2019 Accepted: 6 December 2019

Published online: 27 December 2019

\section{References}

1. Hill AV. The physiological basis of athletic records. Lancet. 1925;206:481-6 Available from: http://www.thelancet.com/journals/a/article/PIIS0140-6736 (01)15546-7/fulltext.

2. Monod H, Scherrer J. The work capacity of a synergic muscular group. Ergonomics. 1965;8:329-38.

3. Ward-Smith AJ. A mathematical theory of running, based on the first law of thermodynamics, and its application to the performance of world-class athletes. J Biomech. 1985;18:337-49.

4. Moritani T, Nagata A, Devries HA, Muro M. Critical power as a measure of physical work capacity and anaerobic threshold. Ergonomics. 1981;24:339-50.

5. Hughson RL, Orok CJ, Staudt LE. A high velocity treadmill running test to assess endurance running potential. Int J Sports Med. 1984;5(01):23-5.

6. Wakayoshi K, Ikuta K, Yoshida T, Udo M, Moritani T, Mutoh Y, et al. Determination and validity of critical velocity as an index of swimming performance in the competitive swimmer. Eur J Appl Physiol Occup Physiol. 1992;64:153-7.

7. Kennedy MD, Bell GJ. A comparison of critical velocity estimates to actual velocities in predicting simulated rowing performance. Can J Appl Physiol. 2000;25:223-35.

8. Williams C, Ratel S, editors. Human muscle fatigue: Routledge; 2009.

9. Bourne MN, Webster KE, Hewett TE. Is fatigue a risk factor for anterior cruciate ligament rupture? Sport Med. 2019; Available from: http://ink. springer.com/10.1007/s40279-019-01134-5.

10. Bigland-Ritchie B, Woods JJ. Changes in muscle contractile properties and neural control during human muscular fatigue. Muscle Nerve. 1984;7:691-9.

11. Vøllestad NK. Measurement of human muscle fatigue. J Neurosci Methods. 1997;74:219-27.

12. Davis MP, Walsh D. Mechanisms of Fatigue. J Support Oncol. 2010;8:164-74.

13. Kay D, Marino FE. Fluid ingestion and exercise hyperthermia: Implications for performance, thermoregulation, metabolism and the development of fatigue. J Sports Sci. 2000;18:71-82.

14. Kay D, Marino E, Cannon J, St A, Gibson C, Noakes TD. Evidence for neuromuscular fatigue during high-intensity cycling in warm , humid conditions. Eur J Appl Physiol. 2001;84:115-21.

15. Abbiss CR, Laursen PB. Models to explain fatigue during prolonged endurance cycling. Sport Med. 2005;35:865-98. 
16. Poole DC, Burnley M, Vanhatalo A, Rossiter HB, Jones AM. Critical power: an important fatigue threshold in exercise physiology. Med Sci Sport Exerc. 2016;48:2320-34

17. Craig JC, Vanhatalo A, Burnley M, Jones AM, Poole DC. Critical power: possibly the most important fatigue threshold in exercise physiology. Muscle Exerc Physiol. 2019:159-81 Available from: https://doi.org/10.1016/ B978-0-12-814593-7.00008-6.

18. Ozyener F, Rossiter HB, Ward SA, Whipp BJ. Influence of exercise intensity on the on- and off-transient kinetics of pulmonary oxygen uptake in humans. J Physiol. 2001;533:891-902.

19. Carter H, Pringle JSM, Jones AM, Doust JH. Oxygen uptake kinetics during treadmill running across exercise intensity domains. Eur J Appl Physiol. 2002:86:347-54.

20. Rose EA, Parfitt G. A quantitative analysis and qualitative explanation of the individual differences in affective responses to prescribed and self-selected exercise intensities. J Sport Exerc Psychol. 2007;29:281-309 Available from: http://www.ncbi.nlm.nih.gov/pubmed/17876968.

21. Hall EE, Ekkekakis P, Petruzzello SJ. The affective beneficence of vigorous exercise revisited. Br J Health Psychol. 2002;7:47-66.

22. Welch AS, Hulley A, Ferguson C, Beauchamp MR. Affective responses of inactive women to a maximal incremental exercise test: a test of the dualmode model. Psychol Sport Exerc. 2007;8:401-23.

23. Beneke R. Methodological aspects of maximal lactate steady stateimplications for performance testing. Eur J Appl Physiol. 2003;89:95-9.

24. Billat $V L$, Sirvent $P, P y ~ G$, Koralsztein JP, Mercier J. The concept of maximal lactate steady state. Sport Med. 2003;33:407-26.

25. Beneke $\mathrm{R}$, Leithauser RM, Ochentel $\mathrm{O}$. Blood lactate diagnostics in exercise testing and training. Int J Sports Physiol Perform. 2011;6:8-24.

26. Pringle JSM, Jones AM. Maximal lactate steady state, critical power and EMG during cycling. Eur J Appl Physiol. 2002;88:214-26.

27. Dekerle J, Baron B, Dupont L, Vanvelcenaher J, Pelayo P. Maximal lactate steady state, respiratory compensation threshold and critical power. Eur J Appl Physiol. 2003;89:281-8.

28. Mattioni Maturana F, Keir DA, McLay KM, Murias JM. Can measures of critical power precisely estimate the maximal metabolic steady-state? Appl Physiol Nutr Metab. 2016:41:1197-203.

29. Keir DA, Fontana FY, Robertson TC, Murias JM, Paterson DH, Kowalchuk JM, et al. Exercise intensity thresholds: identifying the boundaries of sustainable performance. Med Sci Sports Exerc. 2015;47:1932-40.

30. Black MI, Jones AM, Blackwell JR, Bailey SJ, Wylie LJ, McDonagh STJ, et al. Muscle metabolic and neuromuscular determinants of fatigue during cycling in different exercise intensity domains. J Appl Physiol [Internet]. 2017;122:446-59 Available from: http://jap.physiology.org/lookup/doi/1 0.1152/japplphysiol.00942.2016.

31. Jones AM, Burnley M, Black MI, Poole DC, Vanhatalo A. The maximal metabolic steady state: redefining the 'gold standard.'. Physiol Rep. 2019;7:e14098 Available from: https://onlinelibrary.wiley.com/doi/abs/10.14814/phy2.14098.

32. Whipp BJ, Huntsman DJ, Storer TW, Lamarra N, Wasserman K. A constant which determines the duration of tolerance to high-intensity work [abstract]. Fed Proc. 1982;41:1591.

33. Dekerle J, Brickley G, Hammond AJP, Pringle JSM, Carter H. Validity of the two-parameter model in estimating the anaerobic work capacity. Eur J Appl Physiol. 2006;96:257-64.

34. Gaesser GA, Carnevale TJ, Alan G, Walter DO, Womack CJ. Estimation of critical power with nonlinear and linear models. Med Sci Sports Exerc. 1995; 27:1430-8.

35. Hill DW. The criticial power concept. Sports Med. 1993;16:237-54.

36. Housh DJ, Housh TJ, Bauge SM. A methodological consideration for the determination of critical power and anaerobic work capacity. Res Q Exerc Sport. 1990;61:406-9.

37. Driss T, Vandewalle $H$. The measurement of maximal (anaerobic) power output on a cycle ergometer: a critical review. Biomed Res Int. 2013.

38. Morton $\mathrm{RH}$. The critical power and related whole-body bioenergetic models. Eur J Appl Physiol. Springer-Verlag. 2006;96:339-54.

39. Josephson RK. Contraction dynamics and power output of skeletal muscle. Annu Rev Physiol. 1993;55:527-46.

40. Yoshihuku Y, Herzog W. Optimal design parameters of the bicycle-rider system for maximal muscle power output. J Biomech. 1990;23:1069-79.

41. Housh DJ, Housh TJ, Bauge SM. The accuracy of the critical power test for predicting time to exhaustion during cycle ergometry. Ergonomics. 1989;32: 997-1004.
42. Brickley G, Doust J, Williams CA. Physiological responses during exercise to exhaustion at critical power. Eur J Appl Physiol. 2002;88:146-51.

43. McLellan TM, Cheung KS. A comparative evaluation of the individual anaerobic threshold and the critical power. Med Sci Sports Exerc. 1992;24: 543-50.

44. Jenkins DG, Quigley BM. Blood lactate in trained cyclists during cycle ergometry at critical power. Eur J Appl Physiol Occup Physiol. 1990;61: 278-3.

45. Scarborough PA, Smith JC, Talbert SM, Hill DW. Time to exhaustion at the power asymptote in men and women [abstract]. Med Sci Sport Exerc. 1991; 23:S12.

46. Hill DW, Smith JC. Determination of critical power by pulmonary gas exchange. Can J Appl Physiol. 1999;24:74-86.

47. Overend TJ, Cunningham DA, Paterson DH, Smith WDF. Physiological responses of young and elderly men to prolonged exercise at critical power. European J Appl Physiol Occup Physiol. 1992;64:187-93.

48. Poole DC, Ward SA, Gardner GW, Whipp BJ. Metabolic and respiratory profile of the upper limit for prolonged exercise in man. Ergonomics. 1988; 31:1265-79.

49. Hopkins WG, Edmond IM, Hamilton BH, Macfarlane DJ, Ross BH. Relation between power and endurance for treadmill running of short duration. Ergonomics. 1989;32:1565-71.

50. Péronnet $F$, Thibault $G$. Mathematical analysis of running performance and world running records. J Appl Physiol. 1989;67:453-65.

51. Morton RH. Modelling human power and endurance. J Math Biol. 1990;28: 49-64.

52. Morton RH. A 3-parameter critical power model. Ergonomics. 1996;39:611-9.

53. Weyand PG, Lin JE, Bundle MW. Sprint performance-duration relationships are set by the fractional duration of external force application. AJP Regul Integr Comp Physiol [Internet]. 2005;290:R758-65 Available from: http:// ajpregu.physiology.org/cgi/doi/10.1152/ajpregu.00562.2005.

54. Morton $\mathrm{RH}$. A new modelling approach demonstrating the inability to make up for lost time in endurance running events. IMA J Manag Math. 2009;20: 109-20.

55. Bull AJ, Housh TJ, Johnson GO, Perry SR. Effect of mathematical modeling on the estimation of critical power. Med Sci Sports Exerc. 2000;32:526-30.

56. Bergstrom HC, Housh TJ, Zuniga JM, Traylor DA, Lewis RW, Camic CL, et al. Differences among estimates of critical power and anaerobic work capacity derived from five mathematical models and the three-minute all-out test. J Strength Cond Res. 2014;28:592-600 Available from: http://content. wkhealth.com/linkback/openurl?sid=WKPTLP:landingpage\&an=00124278-2 01403000-00002.

57. Clark IE, Murray SR, Pettitt RW. Alternative procedures for the three-minute all-out exercise test. J Strength Cond Res. 2013;27:2104-12.

58. Morton RH. Critical power test for ramp exercise. Eur J Appl Physiol Occup Physiol. 1994;69:435-8.

59. Gaesser GA, Wilson LA. Effects of continuous and interval training on the parameters of the power-endurance time relationship for high-intensity exercise. Int J Sports Med. 1988;9:417-21.

60. Johnson TM, Sexton PJ, Placek AM, Murray SR, Pettitt RW. Reliability analysis of the 3-min all-out exercise test for cycle ergometry. Med Sci Sports Exerc. 2011;43:2375-80.

61. Muniz-Pumares D, Karsten B, Triska C, Glaister M. Methodological approaches and related challenges associated with the determination of critical power and curvature constant. J Strength Cond Res. 2019;33:584-96.

62. Black MI, Durant J, Jones AM, Vanhatalo A. Critical power derived from a 3-min all-out test predicts 16.1-km road time-trial performance. Eur J Sport Sci. 2014; 14:217-23 Available from: https://doi.org/10.1080/17461391.2013.810306.

63. Morton RH, Green S, Bishop D, Jenkins DG. Ramp and constant power trials produce equivalent critical power estimates. Med Sci Sports Exerc. 1997;29: 833-6.

64. Vanhatalo A, Doust JH, Burnley M. Determination of critical power using a 3-min all-out cycling test. Med Sci Sports Exerc. 2007;39:548-55.

65. Bar-Or O. The Wingate anaerobic test an update on methodology, reliability and validity. Sport Med. 1987:4:381-94.

66. Nebelsick-Gullett LJ, Housh TJ, Johnson GO, Bauge SM. A comparison of methods of measuring anaerobic work capacity. Ergonomics. 1988;31: 1413-9.

67. Vandewalle H, Kapitaniak B, Grün S, Raveneau S, Monod H. Comparison between a 30-s all-out test and a time-work test on a cycle ergometer. Eur J Appl Physiol Occup Physiol. 1989;58:375-81. 
68. Vanhatalo A, Doust JH, Burnley M. Robustness of a 3 min all-out cycling test to manipulations of power profile and cadence in humans. Exp Physiol. 2008;93:383-90.

69. Burnley M, Doust JH, Vanhatalo A. A 3-min all-out test to determine peak oxygen uptake and the maximal steady state. Med Sci Sports Exerc. 2006;38: 1995-2003.

70. McClave SA, LeBlanc M, Hawkins SA. Sustainability of critical power determined by a 3-minute all-out test in elite cyclists. J Strength Cond Res. 2011;25:3093-8.

71. Bergstrom HC, Housh TJ, Zuniga JM, Traylor DA, Lewis RW, Camic CL, et al. Responses during exhaustive exercise at critical power determined from the 3-min all-out test. J Sports Sci. 2013;31:537-45.

72. Muniz-Pumares D, Pedlar C, Godfrey R, Glaister M. A comparison of methods to estimate anaerobic capacity: Accumulated oxygen deficit and $W^{\prime}$ during constant and all-out work-rate profiles. J Sports Sci. 2017:35:2357-64.

73. Skiba PF. The kinetics of the work capacity above critical power [dissertation]: University of Exeter; 2014

74. Bishop D, Jenkins DG, Howard A. The critical power function is dependent on the duration of the predictive exercise tests chosen. Int J Sports Med. 1998;19:125-9.

75. Jenkins D, Kretek K, Bishop D. The duration of predicting trials influences time to fatigue at critical power. J Sci Med Sport. 1998.

76. Barker T, Poole DC, Noble ML, Barstow TJ. Human critical power-oxygen uptake relationship at different pedalling frequencies. Exp Physiol. 2006;91: 621-32.

77. Bartram JC, Thewlis D, Martin DT, Norton KI. Predicting critical power in elite cyclists: questioning the validity of the 3-minute all-out test. Int J Sports Physiol Perform. 2017;12:783-7.

78. Kuipers H, Verstappen FTJ, Keizer HA, Geurten P, Van Kranenburg G. Variability of aerobic performance in the laboratory and its physiologic correlates. Int J Sports Med. 1985;6:197-201.

79. Triska C, Karsten B, Heidegger B, Koller-Zeisler B, Prinz B, Nimmerichter $A$, et al. Reliability of the parameters of the power-duration relationship using maximal effort time-trials under laboratory conditions. PLoS One. 2017;12:1-12

80. Martin JC, Milliken DL, Cobb JE, McFadden KL, Coggan AR. Validation of a mathematical model for road cycling power. J Appl Biomech. 1998:14:276-91.

81. Morton RH, Billat LV. The critical power model for intermittent exercise. Eur J Appl Physiol. 2004;91:303-7

82. Ferguson C, Rossiter HB, Whipp BJ, Cathcart AJ, Murgatroyd SR, Ward SA Effect of recovery duration from prior exhaustive exercise on the parameters of the power-duration relationship. J Appl Physiol. 2010;108: 866-74.

83. Skiba PF, Chidnok W, Vanhatalo A, Jones AM. Modeling the expenditure and reconstitution of work capacity above critical power. Med Sci Sports Exerc. 2012:44:1526-32

84. Skiba PF, Clarke D, Vanhatalo A, Jones AM. Validation of a novel intermittent W' model for cycling using field data. Int J Sports Physiol Perform. 2014;9: 900-4.

85. Skiba PF, Fulford J, Clarke DC, Vanhatalo A, Jones AM. Intramuscular determinants of the ability to recover work capacity above critical power. Eur J Appl Physiol. 2015;115:703-13.

86. Skiba PF, Jackman S, Clarke D, Vanhatalo A, Jones AM. Effect of work and recovery durations on $W^{\prime}$ reconstitution during intermittent exercise. Med Sci Sports Exerc. 2014;46:1433-40.

87. Bartram JC, Thewlis D, Martin DT, Norton Kl. Accuracy of $W^{\prime}$ recovery kinetics in high performance cyclists - modelling intermittent work capacity. Int J Sports Physiol Perform. 2018;13:724-8.

88. Bickford P, Sreedhara VSM, Mocko GM, Vahidi A, Hutchison RE. Modeling the expenditure and recovery of anaerobic work capacity in cycling. Proceedings. 2018:219 Available from: http://www.mdpi.com/2504-3 900/2/6/219

89. Nike introduces breaking2: the quest to break the two-hour marathon barrier. Nike News. Available from: https://news.nike.com/news/2-hourmarathon

90. Hoogkamer W. How biomechanical improvements in running economy could break the 2-hour marathon barrier. Sport Med. 2017:47:1739-50.

91. Hoogkamer W, Snyder KL, Arellano CJ. Modeling the benefits of cooperative drafting: is there an optimal strategy to facilitate a sub-2-hour marathon performance? Sport Med. 2018;48:2859-67.
92. Hoogkamer W, Snyder KL, Arellano CJ. Reflecting on Eliud Kipchoge's marathon world record: an update to our model of cooperative drafting and its potential for a sub-2-hour performance. Sport Med. 2019;49:167-70 Available from: https://doi.org/10.1007/s40279-019-01056-2.

93. Whipp BJ, Ward SA, Lamarra N, Davis JA, Wasserman K. Parameters of ventilatory and gas exchange dynamics during exercise. J Appl Physiol. 1982;52:1506-13.

94. Caen K, Bourgois JG, Bourgois G, Van der Stede T, Vermeire K, Boone J. The reconstitution of $W^{\prime}$ depends on both work and recovery characteristics. Med Sci Sports Exerc. 2019;51:1745-51.

95. de Jong J, Fokkink R, Olsder GJ, Schwab A. The individual time trial as an optimal control problem. Proc Inst Mech Eng Part P J Sport Eng Technol. 2017:231:200-6.

96. Burke ER, Pruitt AL. Body positioning for cycling. In: Burke ER, editor. HighTech Cycl. 2nd ed: Human Kinetics; 2003. p. 69-92.

97. Morton RH. Isoperformance curves: an application in team selection. $J$ Sports Sci. 2009:27:1601-5.

98. Halson S, Peake J, Sullivan J. Wearable technology for atlethes: information overload and pseudoscience? Int J Sports Physiol Perform. 2016;11:705-6.

99. Evenson KR, Goto MM, Furberg RD. Systematic review of the validity and reliability of consumer-wearable activity trackers. Int J Behav Nutr Phys Act. 2015:12:159 Available from: https://doi.org/10.1186/s12966-015-0314-1.

100. Ashtiani F, Sreedhara VSM, Vahidi A, Mocko G, Hutchison R. Experimental modeling of cyclists fatigue and recovery dynamics enabling optimal pacing in a time trial: 2019 Am Control Conf. American Automatic Control Council; 2019. p. 5083-8.

101. Novak AR, Dascombe BJ. Agreement of power measures between Garmin Vector and SRM cycle power meters. Meas Phys Educ Exerc Sci. 2016;7841 1-6 Available from: http://www.tandfonline.com/doi/full/10.1080/109136 7X.2016.1191496.

102. Bouillod A, Pinot J, Soto-Romero G, Bertucci W, Grappe F. Validity, Sensitivity, reproducibility, and robustness of the PowerTap, Stages, and Garmin Vector power meters in comparison with the SRM device. Int J Sports Physiol Perform. 2017;12:1023-30.

\section{Publisher's Note}

Springer Nature remains neutral with regard to jurisdictional claims in published maps and institutional affiliations.

\section{Submit your manuscript to a SpringerOpen ${ }^{\circ}$ journal and benefit from:}

- Convenient online submission

- Rigorous peer review

- Open access: articles freely available online

- High visibility within the field

- Retaining the copyright to your article

Submit your next manuscript at $>$ springeropen.com 